\title{
Lymphoedema of the limbs: an experience from a tertiary clinic in the central province of Sri Lanka
}

\author{
P.C.A Ratnatunga, C. Dandeniya, N. Kandappa, N. Balasuriya, E.G Manorangi \\ Department of Surgery, Faculty of Medicine, University of Peradeniya Sri Lanka.
}

Key words: Lymphoedema; limbs; audit

\begin{abstract}
Objective

A descriptive study to evaluate the pattern of presentation of lymphoedema of limbs to a tertiary care clinic in the central province of Sri Lanka.
\end{abstract}

\section{Patients and Method}

Patients with lymphoedema seen over 28 years, (1980-2007) in the vascular clinic at the General Hospital Peradeniya were reviewed retrospectively.

\section{Results}

649 cases of lymphoedema of limbs were seen. 47 were in the upper limb, 36 of whom were secondary, mostly following axillary clearance associated with a mastectomy, and 11 cases were idiopathic.

602 patients had their lower limbs involved, with 96 cases amongst them being secondary, mostly to trauma, filariasis and a few with pelvic carcinoma and lymphoma. The rest $(\mathrm{n}=506)$ were considered to have lymphoedema of primary aetiology. The involvement of the legs was predominantly below the knees. A late onset group of lymphoedema patients, predominantly males above 60 years posed a problem in diagnosis and is worth future study.

Complications among patients with lymphoedema of lower limbs with no overt secondary cause included inter digital cleft sepsis in $54.5 \%$, cellulitis or a history of the same in $66 \%$, lymphangitis $16 \%$ lymphadenitis $3.5 \%$ and $11 \%$ had septicaemia which aggravated the clinical state.

\section{Conclusion}

Primary lymphoedema needs recognition as the dominant

Correspondence: P.C.A Ratnatunga

E-mail: channasurg@yahoo.com

Received: 19-03-2017 Accepted: 02-08-2017

(iD) http://orcid.org/0000-0001-5101-892X

DOI: http://doi.org/10.4038/sljs.v35i2.8381

The Sri Lanka Journal of Surgery 2017; 35(2): 1-6 cause of limb lymphoedema especially in the Central Province. This diagnosis is required to prevent complications which need long term treatment. A filarial aetiology for most cases, as is popularly believed, is not evidence based. Secondary causes for lower limb lymphoedema must be looked for, such as pelvic malignancy and lymphoma. Trauma or surgery over lymphatic pathways should be avoided to prevent limb lympheodema.

\section{Introduction}

No significant documentation exists on the pattern of presentation of lymphoedema in Sri Lanka, though there exists records of its presence as a disease entity since the eighteenth century [1]. Lymphoedema is a disorder that has geographical implications such as possible association to Filaria [2], bare foot walking and the soil texture as in podoconioses [3]. It also involves genetic factors [4], with a pathogenesis seen to be of protean origin.

The complications associated with lymphoedema, namely interdigital cleft sepsis, lymphangitis, lymphadenitis and cellulitis are quite disabling and aggravate the morbidity of the disease [5]. Surgical procedures that have been attempted to rectify lymphedema have yielded poor long- term results [5].

The data regarding prophylactic care given to such limbs is inadequate, and makes it difficult to adopt focused and effective remedial measures. We present a database collected and collated over twenty eight years of attending to such patients in the hope that it will provide a scaffolding for care of this as yet mostly ignored area of clinical care.

\section{Patients and Method}

Over a period of 28 years (1980-2007), 649 patients with limb lymphoedema were seen in the vascular clinic of the General Hospital Peradeniya. Majority of patients were from Central Province of Sri Lanka and their clinical and investigative records were studied retrospectively.

Other causes of limb oedema such as cardiac, hepatic, renal, hypothyroidism, hypo-proteinaemia of varying aetiology and local causes like hypertrophy, angiodysplasia, neurofibroma- 
tosis lipodystrophy, myxoedema venous and inflammatory oedema were eliminated by a careful clinical history, examination and investigation.

Filariasis was suspected as possibly being the cause if either the blood smear for microfilaria, the FAT (filarial antibody titre) or filarial antigen test were positive. The new immunochromatographic test for Wucheria bancrofti antigen was not done due to its non-availability until recently and high cost. This should be stated in the end as a limitation.

Ultrasound examinations of proximal lymphatics and lymph nodes were not routinely carried out due to unavailability during the early years. Duplex scans and isotope scintigrams were done where indicated. Ethical clearance to peruse the records was obtained from the Faculty of Medicine, University of Peradeniya.

\section{Results}

Forty seven patients had upper limb lymphoedema and 602 had lymphoedema of the lower limbs.

\section{Lymphoedema of the upper limb $(n=47)$.}

Thirty six patients had secondary lymphoedema (Table 1) with axillary lymph node clearance in breast cancer being the most common underlying cause. Eleven cases of lymphoedema were idiopathic. Idiopathic upper limb lymphoedema had no antecedent inflammation. The oedema was confined mostly to the subcutaneous tissue of the forearm and the dorsum of the hand. A single limb was involved in all cases and a family history of similar oedema was absent. There were no palpable lymph nodes.

Patent veins were demonstrated initially using a hand held Doppler. Later duplex scans were used when they were available in the unit. The ESR, and WBC/DC were normal. FAT titre, smears for microfilaria $(\mathrm{mf})$ and filaria antigen titre were negative. Isotope scintigrams showed no obstruction in the ipsi-lateral axilla in the two cases in whom it was done. The oedema in all cases subsided spontaneously in few months without any treatment.

\section{Table 1. Causes of Secondary Lymphoedema of} the upper limb (n-47)

\begin{tabular}{|l|l|}
\hline Breast Cancer - Post Mastectomy +/- DXT & $=29$ \\
\hline $\begin{array}{l}\text { Breast Cancer with extensive with nodal } \\
\text { spread }\end{array}$ & $=02$ \\
\hline Malignant Melanoma with nodal metastases & $=01$ \\
\hline Tuberculosis with nodes and sinuses & $=01$ \\
\hline Filariasis & $=03$ \\
\hline Idiopathic & $=11$ \\
\hline
\end{tabular}

* DXT $=$ Irradiation

\section{Secondary lymphoedema}

Forty four were females and 26 males, in those recorded $(n=70 / 95)$. Ten had above knee, 53 below knee $(76 \%)$ and 7 below the lower limbs.

\begin{tabular}{|l|l|l|l|}
\hline \multicolumn{4}{|c|}{$\begin{array}{l}\text { Table 2. Causes of Secondary Lymphoedema of lower } \\
\text { limb ( } \mathbf{n = 9 5} \text { ) }\end{array}$} \\
\hline Trauma & $=31$ & Post-surgery & $=13$ \\
\hline Filariasis & $=22$ & Endometriosis & $=03$ \\
\hline Tuberculosis & $=04$ & Irradiation & $=04$ \\
\hline Eczema & $=05$ & Non-specific & $=02$ \\
\hline Carcinoma in the pelvis & $=07$ & & \\
\hline Lymphoma with +/-DXT & $=04$ & & \\
\hline
\end{tabular}

31 patients had trauma (non-surgical trauma-NST) caused by wounds over the lymphatic pathways, non-healing traumatic wounds of the feet and incision of inguinal node abscesses. The post-surgical causes included patients having persistent oedema with recurrent lymphangiitis following damage during varicose vein stripping and harvesting the saphenous vein for cardiac and arterial bypass surgery. Five patients had long term infected eczema of the legs and feet with lymphadenopathy. The demography of this group was, M: F ratio $7: 15$, and the mean duration to lymphoedema is 8.8 years.

Twenty two were suspected as being due to filarasis. The FAT was positive in all cases but the microfilarial smears done on all were negative. No nematodes were isolated by ultrasound when it was performed during the last few years of the study. $8 / 22$ patients were from Kurunegala 21/22 had below knee swelling and in the other patient the scrotal skin was involved. $8 / 22$ of the patients had bilateral involvement. There were no cases with above knee lymphoedema. The mean duration of lympoedema was 30.18 years

Lymphoedema was thought to be of tuberculous aetiology in four patients with a past history of pulmonary tuberculosis. In all, calcification was seen in para- aortic lymph nodes on X-rays. Both lower limbs were affected, ascending to above knee, lower abdomen and scrotum with time.

Malignancy was found in $11(1.8 \%)$ patients, their mean age being 62 years and mean duration of lymphoedema at presentation was 3 months. Three had carcinoma of the prostate, one carcinoma of the penis with a block dissection of the inguinal nodes, three metastatic pelvic nodes from an unknown primary. In one of the latter, ureteric obstruction and the consequent hydronephrosis focused us to the pelvic nodal pathology. Four patients had lymphomas which included a small cell and a follicular variety in two cases. One of them presented with lymphoedema of recent onset, 20 years after being diagnosed and irradiated at the age of 18 years.

Table 3 compares the age of onset in the dominant secondary 
causes $(n=68 / 95)$ for lymphoedema. The degree of lymphoedema in these secondary cases were, WHO Grade I in 42, II in 22 and III in 04. The duration of those secondary lymphoedema is often short.(Figure 1.)

\begin{tabular}{|c|c|c|c|c|c|}
\hline \multicolumn{6}{|c|}{ Table 3. Age at onset of secondary lymphoedema } \\
\hline Yrs:group & NST & "Filariasis" & ST & Cancer & $\mathbf{n}=$ \\
\hline $0-10$ & 00 & 00 & 01 & 00 & 01 \\
\hline $11-20$ & 04 & 02 & 03 & 00 & 09 \\
\hline $21-30$ & 05 & 05 & 03 & 00 & 13 \\
\hline $31-40$ & 06 & 07 & 01 & 02 & 16 \\
\hline $41-50$ & 07 & 01 & 02 & 01 & 11 \\
\hline $51-60$ & 04 & 03 & 00 & 01 & 08 \\
\hline $61-70$ & 02 & 02 & 00 & 03 & 07 \\
\hline $71-80$ & 00 & 02 & 01 & 00 & 03 \\
\hline
\end{tabular}

$*$ NST $=$ Non Surgical Trauma $*$ ST $=$ Surgical Trauma

In NST and ST the patients were often young at onset $(82 \%$, 50 yrs:or less). See Table 3

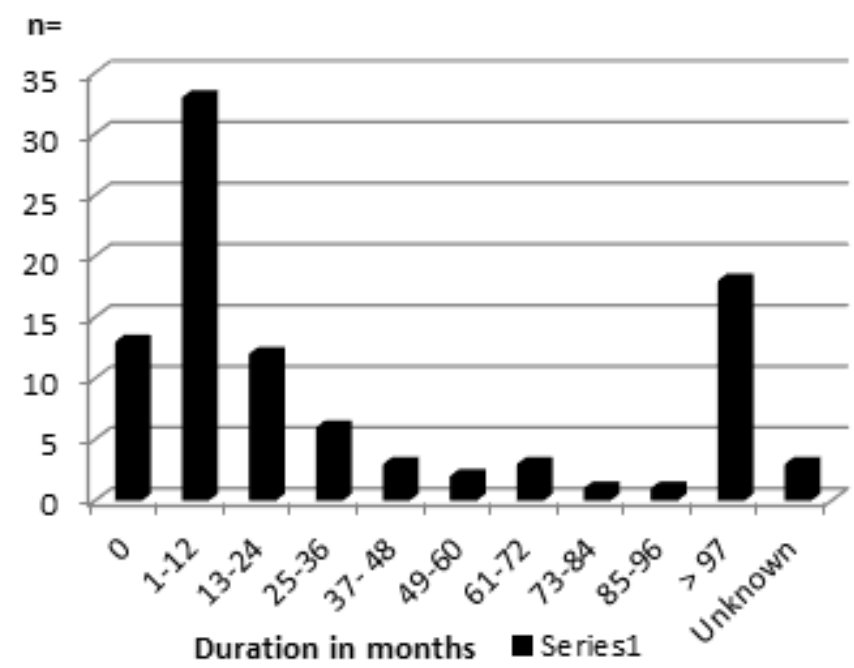

Figure 1. The duration of secondary lymphoedema in lower limbs

Primary lower limb lymphoedema $(n=507)$

This group included patients with familial disease and associated congenital anomalies. The lymphoedema came on early in life (Fig 2) and was dominantly female in the 10 - 40 year group (Fig 3). The short duration of lymphoedema since onset (Fig 4) in those involved and the absence of a secondary cause is likely evidence that it is primary.

The right, left and both lower limbs were involved at presentation in $37.2 \%, 33.3 \%$ and $30.4 \%$ respectively. $14.2 \%$ had above knee lymphoedema, 69.2\% below knee lymphoedema and $13.8 \%$ below ankle lymphoedema. There was a high prevalence of unilateral or bilateral above knee lymphoedema which are features of its possible primary nature.

According to the WHO Grade of lymphoedema, at presentation $\mathrm{n}=463(57.5 \%)$ was grade I, II $36.5 \%$ and III $6 \%$, Figs: $9 \mathrm{a}-\mathrm{d}$. The FAT was negative in all 87 of 507 patients tested.

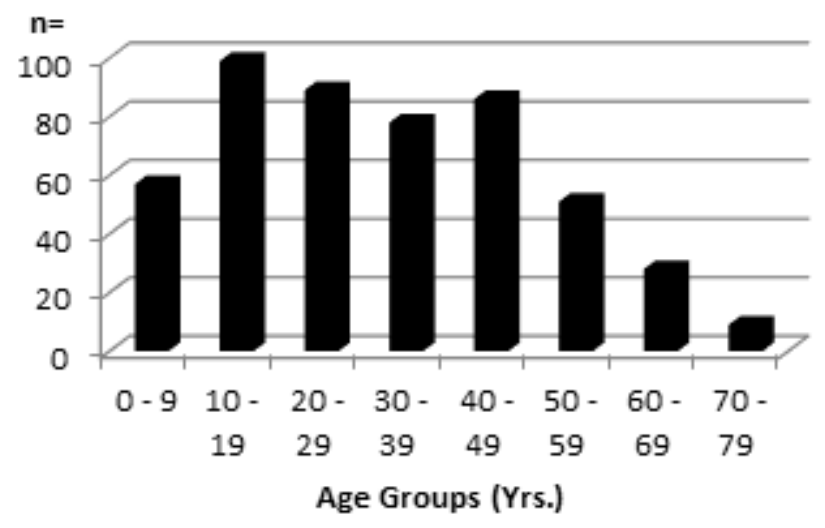

Figure2. Age at onset of primary lower limb Lymphoedema

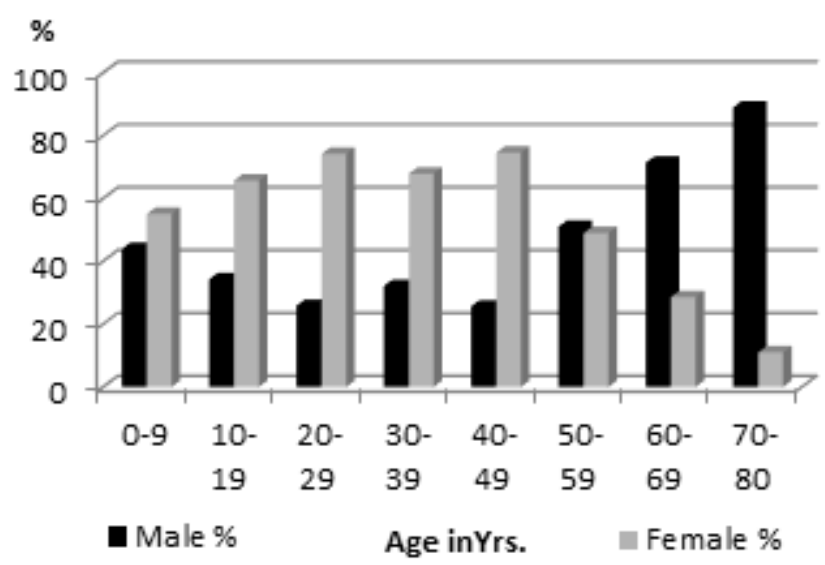

Figure 3. Age at onset of primary lymphoedema of lower limbs Gender \%

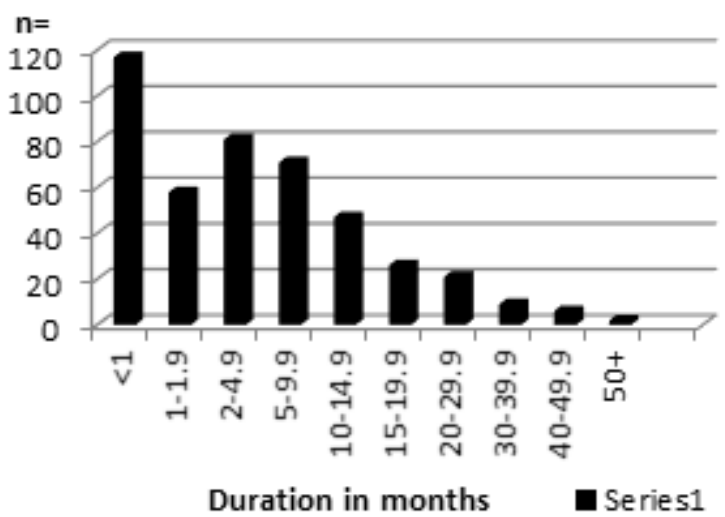

Figure 4. Primary lymphoedema of lower limbs - Duration

\section{Familial Cases}

\section{Patterns of Primary Lymphoedema (Audit n=507)}

Family members were involved in 34 patients. Two of these patients were born with lymphoedema (Milroy's Disease). The relationships with the numbers within parenthesis were, 
mother (9), daughter (4), sister (12), son (2), brother (7), father (7), and others (1).

The associated congenital anomalies observed were, Milroy's disease 2, Turner syndrome 1, Klippel Trenaunay Syndrome 1, Congenital ptosis 1, Yellow Nail Syndrome 1, Distichiaisis 1.

\begin{tabular}{|l|l|c|c|}
\hline \multicolumn{4}{|c|}{ Table 4. Primary Lymphoedema of Lower Limbs } \\
(Audit n= 507) \\
\hline Classification & Age in Yrs. & $\mathbf{n}=$ & M:F \\
\hline \multirow{3}{*}{ Congenital } & At birth & 34 & $1: 1.13$ \\
\cline { 2 - 4 } & $1-1.9$ & 6 & $1: 2$ \\
\cline { 2 - 4 } & $2-14$ & 45 & $1: 1.6$ \\
\hline Precox & $14-35$ & 218 & $1: 2.4$ \\
\hline Tarda & $>35$ & 204 & $1: 1.79$ \\
\hline
\end{tabular}

The onset of lymphoedema had gender equality in the $<9$ year age group (see fig 3), followed by female dominance (75\%) in the middle years (10-49) as would be expected of a primary cause. The M: F ratio overall was 1:1.72.

The patterns of primary lymphoedema is shown in Table 4. The "tarda" group especially those over 40 years at the age of onset, $(n=174) \quad$ constituted $34.4 \%$ of patients. There was a female dominance in the 40 - 60 year group $(65 \%)$ but a male dominance in those over 60 years) (Fig 3) this male dominance accounted for 28 males.

The percentage with bilateral involvement was seen in 19\% in those over 50 years, and $23 \%$ in those over 60 years. At onset $97 \%$ were involved below knee.

The duration of lymphoedema at presentation in those recorded, $(n=441 / 507)$ shows that in $40 \%$, it is of less than 2 years duration (Fig 4). Fig: 5 shows that there is no definite evidence that above knee lymphoedema ascends with

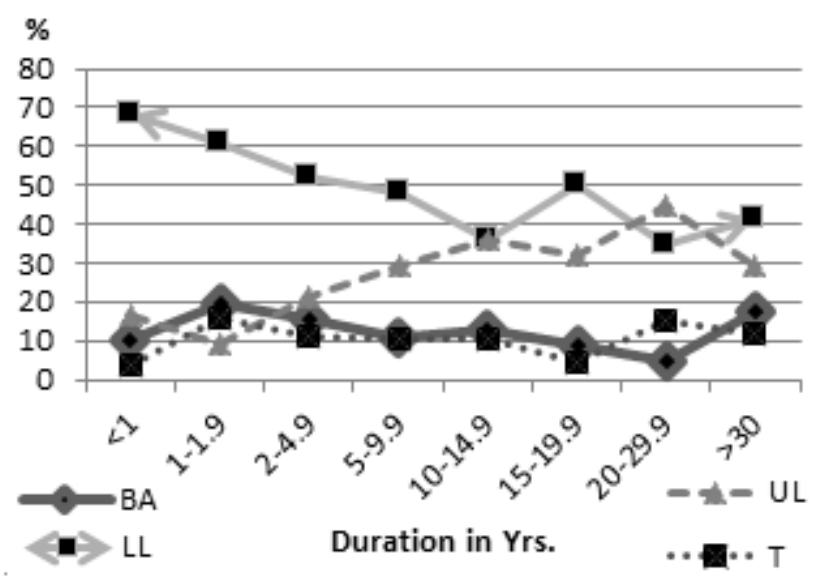

Figure 5. Extent of lymphoedema related to duration. BA - below ankle LL - lower leg UL - upper leg T - thigh increasing duration. Those with below knee lymphoedema seem to ascend from lower leg to upper with time.

The complications in those with primary lower limb lymphoedema.

Interdigital cleft sepsis (tinea pedis) was found on $54.5 \%$ of patients with "primary" lymphoedema on admission. The prevalence was as high as $72 \%$ if the patient had a less than a year's history on admission. Thereafter the prevalence regressed to 60,65 , and 63 , to $50 \%$ over the cohorts admitted 5 - 10, 10 - 15.15 - 20, and 40 - 50 years duration respectively, prior to be seen by us. $30 \%$ of the cases were associated with cellulitis on admission and another $33 \%$ had a history suggestive of cellulitis. Bacterial cultures often grew Streptococci. $16 \%$ had lymphangitis and $3.5 \%$ of associated ipsilateral inguinal lymphadenitis were seen to follow these attacks .Ulceration of the lymphoedema, also was seen. 57 patients (11\%) having fever, and of whom, 5 were accompanied with severe chills and rigors, were diagnosed as having septicaemia on admission.

15 patients with grade III lymphoedema, with skin changes, with or without ulceration, whose infection was controlled and who wished to undergo cosmetic surgery, were operated on with a Modified Thompson Procedure, with fair (patient satisfying) results.

\section{Discussion}

Idiopathic upper limb lymphoedema is an enigma. Aetiological possibilities include lymphatic overload due to allergy [6]. It is a self-limiting clinical condition.

It is noteworthy that $76.6 \%$ had a secondary cause in the upper limb group, while a secondary cause was detected in only 15.8 $\%$ of the lower limb group. It is a common observation that lymphoedema of the toes feet and legs go often unnoticed, leading to inter digital sepsis aggravating the clinical state [7, 8].

Filaria was suspected in only 22 cases. The mere demonstration of exposure to filaria by a positive FAT does not give validity to the claim that filaria is the aetiology in most cases of lymphoedema, at least in the central province. Data on the prevalence of FAT (Ig4) titers in the central province during the period of study is not available. Hence the significance of a positive result cannot be deduced. Had we done antigen studies, especially in "non-endemic" Kandy we might have staked a claim if it was positive. However Kurunegala in the North Western Province (8/22), showed a high prevalence in our study and lends weight to the possibility of its existence as an endemic filarial pocket. The below knee lymphoedema in almost all filaral cases $(n=21 / 22)$ is compatible with similar findings in Indian [9] and African studies [10]. Unless a cofactor exists such as secondary bacterial sepsis, above knee 
lymphoedema as a manifestation of lymphatic filariasis is unlikely to take place [2]. It's localization to below knee lymphoedema is explicable based on a block at the sentinel node for the leg [11]. Unless the filarial nematode is isolated by ultrasound within the lymphatic trunks or seen in node biopsies, it would be difficult to confirm its causative role in lymphoedema [2]. The pathogenesis of the lymphangiectasia associated in the lymphatics distal to live adult Wuchereria Bancrofti filarial worms are not now considered obstructive but thought to be due to lymphatic dysfunction caused by substances released by live adult worms [2]. This dysfunction is not seen to revert even after the death of the worm by diethyl carbamazine [12]. That we did not observe live or dead worms in this study reinforces the reality that filarial causation is a myth and is not the dominant aetiology, at least among those with lymphoedema in the Central Province of Sri Lanka.

Anti-filarial drugs are not effective in chronic lymphoedema. Current pathology suggests that the block is caused by secondary sepsis. Further their indiscriminate use shows uncritical acceptance of a filarial aetiology and is a commonplace practice in non- endemic areas of Sri Lanka. It is an unwarranted practice.

In the patient group with secondary causes, the recurrent bouts of lymphangitis and para lymphangitis would leave on resolution, a distortion, both internally and externally, of these $1 \mathrm{~mm}$ bore tubes that form lymphatic trunks. The nodal architecture especially in the sentinel nodes (lower-most in the vertical set [11]) would be distorted and on occasion be fibrosed [5]. Hence possibly the high prevalence (88\%) of below knee lymphoedema in this group, as is a feature in other international series [11]. Cellulitis is a common sequel that aggaravtes the morbidity. Bacterial entry into the subcutaneous tissue in those with skin changes is well documented [13].

Though malignancy constitutes only $11 \%$ of this secondary group, they must be diagnosed early and hence a comprehensive examination of patients with lymphoedema is mandatory. A careful but relevant history, with a careful examination, looking for pelvic malignancy is a must. This must be followed by a pelvic ultrasound examination. A hydronephrotic kidney maybe the only clue of ureteric compression by a mass of metastatic lymph nodes of a pelvic malignancy as was seen in one of our cases. Further imaging is a must in suspicious cases, especially those who are elderly and have a short duration lymphoedema which is grade I. Prostatic carcinoma was the dominant pelvic malignancy in our study, as has been in international series [14].

Lower limb Lymphoedema that did not show features suggestive of a secondary aetiology were considered to be primary. They showed the classical presentation of this group, namely the equal prevalence of genders in the congenital group, and the dominance of females in the precox group, as in other international series [7, 16]. In most international studies the tarda group in primary lymphoedema constitutes usually $10-15 \%$ of cases [15]. In our study $40.3 \%$ of patients based on the age of onset were in the $35+$ year group.

In the $60+$ and $70+y r s$. age groups $(n=37)$, males dominate and constitute 28 patients (75\%). Fig: 3 . This stresses the importance of investigating for malignancy of the prostate prior to assigning aetiology to a primary cause. This could also be contributed to by the greater exposure of cracked feet to hot silca of the soils $[2,3,5]$ i.e pseudoconioses, being more common among working males. There being bilateral involvement in $23 \%$ of this group and the $97 \%$ below knee affliction supports this possibility. This warrants further study.

The presence of a large number of lymphoedema patients with what is assumed to be of Primary origin, i.e. 506/602 (84\%), more than quoted in most international series, $60 \%$ [7], $45 \%$ [17], 62\% [14], needs explanation. This may be due to most primary cases gravitating to our clinic being the only one in the central province, or maybe there could be some filarial cases unwittingly included in the primary group as we did not do a FAT on all cases. The large number of $>50$ years at onset may also be contributory.

The frequency of infective attacks on their lymphatic system warrants highlighting, often originating in the frequent trichophytosis in their interdigital clefts and ensuing secondary infections between the swollen toes. Sometimes the leg remains clinically warm because of sub clinical sepsis and there could be a direct relationship between the numbers of attacks of sepsis, the duration of the illness with eventually the grade of the lymphoedema However a statistically significant correlation of these factors could not be elicited.

\section{Conclusion}

Lymphoedema is not an uncommon clinical condition, but has unfortunately been not given due consideration by the medical community in Sri Lanka, despite the morbidity, the psychological upset and the handicaps it causes. The limitations of this study include that it is a retrospective study; the lack of corroborative ultrasound or duplex in all patients; assumption of a filarial aetiology based on a positive FAT whose specificity is at best doubtful; the assumption that where a secondary cause was not found it was primary in aetiology. In the future we would like to improve our data base by studying the titre of filarial antigens on all patients and more carefully assess the prostate with ultrasound and add a routine PSA to the assessment of our senior patients with lymphoedema. 
All authors disclose no conflict of interest. The study was conducted in accordance with the ethical standards of the relevant institutional or national ethics committee and the Helsinki Declaration of 1975, as revised in 2000 .

\section{References}

1. Thosmasz.H.G.Elephantiasis arabum and its treatment, Journal of the Ceylon branch of the British Medical Association 1888, 2, $1-5$.

2. G,Dreyer,J, Noroes, J.Figueredo-Silva and W,F.Piessens. Pathogenesis of Lymphatic Disease in Bancroftian Filariasis. Parasitol Today 2000.16 544-548.

PMID: 3515791

3. Price E. W. The pathology of non - filarial elephantiasis of the lower legs. Trans R Soc Trop Med Hyg 1972, 66, 150- 159 https://doi.org/10.1016/0035-9203 (72) 90063-6

4. Milroy W.F. An undescribed variety of hereditary oedema. New York Medical Journal 1892, 56, 505-508.

5. N. Browse, K.cases. G.Burnand. Mortimer P.S. Diseases of the Lymphatics 1st ed, London Arnold 2003

6. Sing H.Gomez C., Calonje E, Browse N.L. Lymphangiomatosis of the limbs clinic-pathologic and analysis of a series with a good prognosis. Journal of Surgical Pathology1995, 10, 125-133. PMID: 7832272

7. Allen E.V., Ghormley R.K. Lymphoedema of the extremities: etiology, classification and treatment; report of 300 cases Arch Intern Med 1935,9,516-539. http://dx.doi.org/10.1016/S0002-8703(36)90404-0

8. Moffatt J, Franks P,J, Doherty D, J, Williams A. F, Badger C. Jeffs, E. Bosanquet N. Mortimer P.S Lymphoedema: an underestimated health problem. Quarterly Journal of Medicine,2003,96.731-738. https://doi.org/10.1093/qjmed/hcg126

9. Dondero T.J., Battacharaya N.C., Black H.R. Chowdhry A.B. Gubler D.J, Inui T.S, Mukerjee M. Clinical manifestations of Bancroftian Filariasis in a suburb of Calcutta, India Am J Trop
MedHyg 1976,25 64-72.

https://doi.org/10.4269/ajtmh.1976.25.64

PMID:769575

10.Dunyo S.K, Appawu M.,Nkrumah F.K, Baffoe-Wilmot A.,Pedersen E.M, Simonsen P.E. Lymphatic Filariasis on the coast of Ghana. Transactions of the Royal Society of Tropical Medicine and Hygiene, 1996, 90,634-638.

https://doi.org/10.1016/S0035-9203(96)90414-9

11. Ngu A. Konstam P..Chronic lymphoedema in Western Nigeria $\mathrm{Br}$ JSurg, 1964.51,110.

PMID: 14117765

12. Singh G. Raksha, Urehekar A.D. Advanced Techniques for Detection of Filariasis- A Review. International Journal Research Studies in Biosciences.2013,1,17-22.

13.Olzewski W.L, Jamal S.,ManokaranS, Pani S .Kumaraswami V .Kubicka U. Lukomska B.Dworczynski A. Swoboda E. Mikolajczyk F M .Bacteriologic studies of skin, tissue fluid, lymph nodes in patients with Filarial Lymphoedema Am J Trop Med Hyg 1997.57,7-15

PMID: 9242310

14. Smith R.D,Spittell J.A., Schirger A. Secondary lymphoedema its characteristics and diagnostic complications. JAMA,185,80. doi:10.1001/jama.1963.03060020040018

15. Young J.R., The Swollen Leg, Clinical Significance and Differential Diagnosis, Cardiology Clincs 1991,9,443-456. PMID: 1913726

16.Stone,J,E, Hugo N.E. Lymphoedema a Collective review. Surgery Gynecol Obstet 1972,135,625-631 PMID:4562125

17. Cockett D.J.Lymphatic anatomy and lymphoedema Br J Plast Surg 1963, 12-25

PMID: 14256821 